\title{
Homoeopathy for delayed onset muscle soreness: a randomised double blind placebo controlled trial
}

\author{
Andrew J Vickers, Peter Fisher, Claire Smith, Sheena E Wyllie, George T Lewith
}

Research Council for Complementary Medicine, 60 Great Ormond Street, London WC1N 3JF, United Kingdom and Division of Public Health and Primary Care, University of Oxford, Gibson Building, Radcliffe Infirmary, Oxford OX2 6HE, United Kingdom A J Vickers

\section{Royal London \\ Homoeopathic Hospital NHS Trust P Fisher S E Wyllie}

Research Council for Complementary Medicine

C Smith

School of Medicine, University of Southampton G T Lewith

Correspondence to: A J Vickers.

Accepted for publication 26 June 1997

\begin{abstract}
Objective-To pilot a model for determining whether a homoeopathic medicine is superior to placebo for delayed onset muscle soreness (DOMS).

Design-Randomised double blind placebo controlled trial.

Setting-Physiotherapy department of a homoeopathic hospital.

Subjects-Sixty eight healthy volunteers (average age $30 ; 41 \%$ men) undertook a 10 minute period of bench stepping carrying a small weight and were randomised to a homoeopathic medicine or placebo.

Outcome measures-Mean muscle soreness in the five day period after the exercise test, symptom free days, maximum soreness score, days to no soreness, days on medication.

Results-The difference between group means was 0.17 in favour of placebo with $95 \%$ confidence intervals \pm 0.50 . Similar results were found for other outcome measures.

Conclusion-The study did not find benefit of the homoeopathic remedy in DOMS. Bench stepping may not be an appropriate model to evaluate the effects of a treatment on DOMS because of wide variation between subject soreness scores. (Br f Sports Med 1997;31:304-307)
\end{abstract}

Keywords: homoeopathy; muscle soreness; exercise; randomised controlled trial

Delayed onset muscle soreness (DOMS) is a sensation of pain or discomfort that follows unaccustomed muscular exertion. DOMS is usually first noticed 12 hours after exercise, peaks at 36-48 hours, and lasts for some five to seven days in total. Although the cause of DOMS is not fully understood, reversible damage to muscle fibres is thought to be implicated. ' Symptoms are believed to be most severe after eccentric exercise, ${ }^{2}$ where a muscle lengthens under tension, but are susceptible to a training effect such that the degree of soreness after repeated bouts of specific exercise diminishes over time. ${ }^{1}$

Homoeopathy is a form of therapy based on the principle of "let like be cured with like". Practitioners prescribe in a low dose whatever drug would cause symptoms similar to those in the patient were that drug to be taken in a high dose. One well known feature of homoeopathy is that many of the medicines used are so dilute that they are unlikely to contain a single molecule of the original drug. Although often described as an "alternative" or "complementary" therapy, homoeopathy is practised by about 1000 doctors in the United Kingdom and is available on the NHS at five homoeopathic hospitals (source: Faculty of Homeopathy, Powis Place, London WC1).

Practitioners of homoeopathy claim to be able to provide symptomatic relief for DOMS. This claim seems worthy of evaluation, especially as homoeopathy is a simple inexpensive intervention that seems to be free from adverse effects. Moreover, there is preliminary evidence that homoeopathy is of benefit for DOMS. Hildebrandt and Eltze ${ }^{3}$ undertook a series of experiments comparing a homoeopathic medicine known as Rhus tox with placebo for DOMS induced by an hour's exercise of the upper arms. Although significant differences between homoeopathy and placebo are reported for some outcomes in some of these trials, problems with the study include lack of randomisation, inadequate details of baseline characteristics, small numbers of subjects, and multiple hypothesis testing. In a double blind trial, Tveiten et $a l^{4}$ randomised 36 runners to receive homoeopathic arnica or placebo after a marathon. Stiffness scores and blood creatine kinase were lower in the group that received arnica, with differences for soreness approaching statistical significance $(P=0.07)$ on day 2 and 3. No differences were found in levels of haptoglobin, magnesium or enzymes other than creatine kinase.

The research question posed here is: can a homoeopathic medicine reduce levels of DOMS more than placebo? The first task in answering this question was to pilot a suitable model of DOMS induction and assessment. For this study, we aimed to induce DOMS by a simple bench stepping exercise, randomise a small number of subjects to the homoeopathic medicine or placebo on a double blind basis, and measure outcome using a Likert scale. Our main objectives were to determine the appropriateness of the study model and to produce some estimates on which to base a sample size calculation for a definitive trial.

The choice of test medicine was determined by discussion with a number of homoeopaths. Arnica was chosen as it is the classic remedy for any type of soft tissue trauma, Rhus tox because it is indicated for stiffness that worsens on rest but is relieved by exercise, a feature characteristic of DOMS, and sarcolactic acid as it is 
indicated for sore and aching muscles. The most appropriate homoeopathic dose was thought to be a " 30 c potency", where the drugs are prepared by a process of serial dilution and sucussion (agitation) to a theoretical dilution of $10^{-60}$.

They are therefore highly unlikely to contain a single molecule of the original medicine.

While developing the protocol for the study, a group of researchers at the Department of Medicine at Southampton University took an interest in our work and agreed to pilot a similar trial. This was completed just as our study started and its results have been reported elsewhere. ${ }^{5}$ We intended to undertake an individual patient data meta-analysis of both trials to increase the precision of our results.

\section{Methods}

Healthy volunteers aged 18-40 were recruited primarily from staff and visiting health professionals at the Royal London Homoeopathic Hospital. Recruitment took place between April and October 1996. The trial was stopped when a fund to pay volunteers a small fee had been expended.

Subjects were excluded from the trial if they had concurrent musculoskeletal or fatigue disorder, a history of cardiovascular disease, nonsteroidal anti-inflammatory treatment in the week preceding the trial, steroids or "constitutional" homoeopathic treatment by mouth in the previous six months or regularly participated in vigorous exercise in the previous six months. Vigorous exercise was defined as playing a sport, such as squash, tennis, or football, or undertaking an activity designed to increase physical fitness, such as jogging, aerobics, step training, or swimming. Regular participation was defined as undertaking the activity three or more times a week for more than three consecutive weeks and for more than 15 minutes at a time. During the trial, subjects were asked to avoid anti-inflammatory drugs, pain relieving ointments or creams, exercise, stretching or massage. Subjects were given an information leaflet that described the trial. They then provided written informed consent.

Baseline data included age, sex, weight, lower leg length, and level of exercise. The latter was assessed using the following two questions: "do you participate regularly in any activity likely to exercise the legs-for example, cycling, dancing-for more than 10 minutes more than twice a week or has there been a period of more than three consecutive weeks in which you have done so in the previous six months?" and "do you participate in any sport or any activity specifically designed to increase fitness or has there been a period of more than three consecutive weeks in which you have done so in the previous six months?" If a subject gave a positive answer to either of these questions, they were categorised as a moderate exerciser.

Subjects were assigned the next code number available from a sequentially numbered list of randomised treatment allocations and given the appropriately coded bottle of medication. They were instructed to take one tablet by mouth three times a day, starting 24 hours before the bench stepping exercise and stopping when they no longer felt sore.

A homoeopathic complex of Arnica 30c, Rhus tox 30c, and sarcolactic acid 30c was produced by Ainsworth's, a registered homoeopathic manufacturing pharmacy. The medicines were freshly prepared following the guidelines of the Blackie Foundation, a homoeopathic research organisation. Placebos were also prepared that were indistinguishable from the active medication in appearance, taste, or smell. A computer generated randomisation list in permuted blocks of four was given to the manufacturer who dispensed the placebos and active medicine into the coded bottles. The investigator dispensing the medication (SW) was provided with the bottles but had no access to the randomisation list, ensuring that treatment allocation was fully concealed.

The exercise regimen used was that described by Hasson et $a l^{6}$ Subjects stepped up on a bench set at $110 \%$ of their lower leg length while carrying a load equal to $10 \%$ of their body weight. Exercise was continued for 10 minutes at a rate of one step per second. The order of steps was right leg up, left leg up, left leg down, right leg down. This regimen causes greatest soreness in the right thigh and left calf. If the subject failed to complete the exercise test, this was recorded.

After the bench stepping exercise, each subject was given an outcome form, which they were asked to complete at specified times every 12 hours for the next five days. The outcome form consisted of a Likert scale as described by High et al' (see fig 1) and a check box asking whether the subject had stopped taking medication. After the five day period, subjects were asked if they had used any analgesics,

Please tick the sentence below that best describes your level of muscle soreness over the past 12 hours.

[ ] $]_{0}$ A complete absence of soreness

[]$_{1}$ A light pain felt only when touched/a vague ache

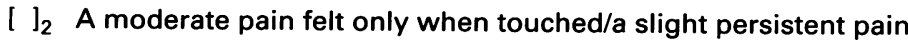

[]$_{3}$ A light pain when walking up or down stairs

[]$_{4}$ A light pain when walking on a flat surface/painful

[]$_{5}$ A moderate pain, stiffness or weakness when walking/very painful

[]$_{6}$ A severe pain that limits my ability to move

Figure 1 Likert scale of muscle soreness (taken from High et al ${ }^{\text {) }}$. 
Table 1 Baseline characteristics. Results are mean (SE)

\begin{tabular}{lll}
\hline Variable & Placebo $(n=28)$ & Homoeopathy $(n=29)$ \\
\hline Age (years) & $30.4(1.11)$ & $30.9(1.01)$ \\
Male/female ratio & $12: 15^{\star}$ & $11: 18$ \\
Weight $(\mathrm{kg})$ & $65.1(2.05)$ & $64.9(2.52)$ \\
Lower leg measurement $(\mathrm{cm})$ & $47.0(1.18)$ & $47.8(0.953)$ \\
Estimate of body mass index & $25.0(1.36)$ & $23.5(1.02)$ \\
Moderate exercisers & $10(35.7 \%)$ & $10(34.4 \%)$ \\
\hline
\end{tabular}

^ One missing observation.

Table 2 Outcome variables. Results are either mean (SD) or median (interquartile range)

\begin{tabular}{lll}
\hline Variable & Placebo $(n=28)$ & Homoeopathy $(n=29)$ \\
\hline Mean five day muscle soreness & $2.21(0.91)$ & $2.38(0.97)$ \\
Days with score 1 or less & $2(1-2.75)$ & $2(1.5-2.5)$ \\
Maximum soreness score & $5(4-5)$ & $5(3-5)$ \\
Days to score of 1 or less & $3.5(3-4.5)$ & $4(3-4)$ \\
Days on medication & $4.5(3-5)^{\star}$ & $4.75(3.5-5)$ \\
\hline
\end{tabular}

* One missing observation. subjects would have been eligible for entry to the current study. Half of the 50 participants the exercise test. There is no reason to believe statistical software package STATA (Stata Corporation, College Station, TX 77840, USA).

An individual patient data meta-analysis was conducted by combining data from the earlier study $^{6}$ with those from the present trial. We felt this was justified on the grounds that the earlier study involved an identical model of muscle soreness and a very similar medicine (Arnica and Rhus tox 30c). The only major difference between the two trials concerned the entry criteria, there being no exclusion on grounds of level of exercise in the first study. However, data on levels of exercise were recorded at baseline and we used this to determine which were thus categorised as "vigorous exercisers" and these were excluded. The remainder were entered into the meta-analysis.

Ethical approval for the trial was provided by the joint University College London/University College London Hospital Committees on the Ethics of Human Research.

\section{Results}

Sixty eight subjects were randomised, of whom 58 undertook the exercise test. Of the remaining 10, three changed their minds after entry into the trial, five did not present for the exercise test, and two broke protocol by receiving homoeopathic treatment. One subject in the placebo group failed to return an outcome form. Table 1 gives the baseline characteristics of the sample. Differences between groups were minor. The number of protocol violations were also similar between groups: five subjects in the active group and four in the placebo group undertook exercise during the five day follow up period; one subject in the active group failed to complete the full 10 minutes of that blinding was broken at any point during the trial.

Table 2 shows outcome variables. Only the main outcome measure, mean muscle soreness, showed evidence of a normal distribution. The difference between group means is 0.17 points on the Likert scale in favour of placebo with $95 \%$ confidence intervals \pm 0.50 . In other words, our results are consistent with homoeopathy being superior by about $15 \%$ and placebo being superior by about $30 \%$.

There is therefore no evidence of a difference between groups. Values of secondary outcome measures were similar in both groups, and no difference approached statistical significance ( $\mathrm{P}>0.5$ for all comparisons).

Analysis of the possible effects of baseline variables on outcome was made difficult by the small numbers. However, a variety of exploratory analyses including correlation and subgroup analyses were carried out. On a simple univariate model (table 3), mean soreness score in the 20 subjects who undertook moderate exercise was lower than in the 37 sedentary subjects $(t=3.22, \mathrm{P}<0.002)$. No other baseline variable was prognostic of outcome.

Nine subjects reported adverse effects. In three cases, all of which were in the placebo

Table 3 Muscle soreness by degree of exercise

$c$

\begin{tabular}{lll}
\hline & $\begin{array}{l}\text { Mean muscle } \\
\text { soreness }\end{array}$ & $S D$ \\
\hline No exercise $(\mathrm{n}=37)$ & $2.57^{\star}$ & 0.79 \\
Moderate exercise $(\mathrm{n}=20)$ & $1.80^{\star}$ & 1.00 \\
\hline $\mathrm{P}^{\star}<0.002$ by ${ }^{\star}$ test &
\end{tabular}

$$
\star \mathrm{P}<0.002 \text { by } t \text { test. }
$$

Table 4 Results of individual patient data meta-analysis

\begin{tabular}{lll}
\hline & Mean muscle soreness & $S D$ \\
\hline Homoeopathy $(\mathrm{n}=44)$ & $2.13^{\star}$ & 1.03 \\
Placebo $(\mathrm{n}=38)$ & $2.20^{\star}$ & 1.06 \\
\hline
\end{tabular}

^ No significant difference between groups. 
Table 5 Results from meta-analysis by type of outcome variable

\begin{tabular}{llll}
\hline & Placebo & Homoeopathy & Relative difference \\
\hline Mean soreness & 2.20 & 2.13 & 0.032 \\
Days soreness score $\leqslant 1$ & 2.25 & 2.45 & 0.089 \\
Days on medication & 4.31 & 4.24 & 0.016 \\
\hline
\end{tabular}

group, subjects stated that it was unlikely that the symptoms were related to the study medication. The symptoms were: a slight throat infection for three days (which had started before taking pills); diarrhoea and stomach upset; sleepiness and very light nausea. Of the remaining six cases, four were in the homoeopathy group and of these, three reported similar symptoms. One subject "woke up with sore muscles on the day of the trial"; another "felt unbalanced, kept walking into objects"; the third reported a "real sense of heaviness". Nausea was present in all three cases. The other three adverse effects reported were dissimilar: constipation (homoeopathy), dry lips (placebo), and a "change in my state of awareness" (placebo) and it seems unlikely that they were caused by the study medication.

The individual patient data meta-analysis failed to change the conclusion of no difference between groups (table 4). Homoeopathy is slightly favoured in the combined analysis with a difference between means of 0.07 points on the Likert scale $(95 \%$ confidence intervals \pm 0.46).

The results of the meta-analysis also justify our methodology of adding scores from Likert scales. Strictly speaking, it is statistically incorrect to add scores from an ordinal outcome measure on the grounds that a linear relation cannot be assumed between different points on the scale. However, as shown in table 5 , the relative difference between groups for the continuous variables of days with a symptom of one or less and days on medication were similar to the difference between groups for summed ordinal scores (mean five day soreness). Treating the Likert scale of muscle soreness as a continuous measure is therefore a justifiable approximation.

\section{Discussion}

We used the data from the meta-analysis to undertake a sample size calculation for a definitive trial. Mean muscle soreness was used as our primary outcome measure. We assumed that the mean soreness score in the placebo group would be as reported in table 4 but that the mean soreness score for the homoeopathy group would be 1.80 . This difference between groups is clinically significant and falls within the $95 \%$ confidence intervals estimated in the meta-analysis. Standard deviatioris were held constant. Setting alpha at $5 \%$ and power at $90 \%$, we calculated that 144 evaluable subjects per group would be required, a total sample size of about 300
Given this figure of 300 , we believe that bench stepping may not be an appropriate model for testing the effects of homoeopathic medicines on DOMS. We found it difficult to recruit suitable volunteers, something that surprised us because the exercise test was relatively short and the degree of discomfort moderate. It took a considerable amount of time and effort to recruit the 57 evaluable subjects and we do not currently consider it feasible to mount a trial with a sample size five times as great.

The way forward may be to assess the effects of homoeopathy on DOMS in long distance runners. Soreness does not have to be specially induced in this group, and the large numbers of runners regularly taking part in races will make it easier to obtain a sufficient sample size. Using a more naturalistic model will also make the results of the trial more applicable to the real world. We have recently randomised the first subjects in such a study. It is expected that the results will be reported in 1998 .

Although it is wise to avoid using pilot studies to test hypotheses, we believe that it would be worth drawing the following conclusions: (a) the trial did not provide evidence for an effect of a homoeopathic medicine on DOMS; (b) we can exclude the possibility that the homoeopathic medicine reduces DOMS by more than $25 \%$ compared with placebo; (c) the great variation in soreness scores after bench stepping suggests that this may not be an appropriate model to evaluate the therapeutic effects of a treatment on DOMS; (d) individuals who undertake moderate exercise experience lower levels of DOMS after a standard exercise test than sedentary subjects.

We would like to thank Ainsworth's homoeopathic pharmacy for providing the medicines and the Dove Healing Trust for donation towards covering the costs of the volunteers.

1 Byrnes WC, Clarkson PM. Delayed onset muscle soreness and training. Clin Sports Med 1986;5:605-14

2 Newham DJ, McPhail G, Mills KR, Edward RH. Ultrastructural changes after concentric and eccentric contractions of human muscle. F Neurol Sci 1983;61:109-22.

3 Hildebrandt G, Eltze C. Uber die Wirksamkeit eine Behandlung des Muskelkaters mit Rhus toxicodendron D4. In: Hutelandgesellschaft für Gesamtmedizin. Wissenschaftliches Archiv der Hutelandgesellschaft für Gesamtmedizin. Vol 1. Heidelberg: Kark F Haug, 1983.

4 Tveiten D, Bruset S, Borchgrevnink CF, Lohne K. Effect of Arnica D 30 after hard physical exercise: a double blind randomized control study. Tidsskr Nor Laegeforen 1991;11: randomized

5 Jawara N, Lewith GT, Vickers AJ, Mullee M, Smith C. Homoeopathic arnica and rhus toxicodendron for delayed onset muscle soreness: a pilot for a randomised, doubleblind, placebo-controlled trial. British Homoeopathic fourna 1997;86:10-5.

6 Hasson S, Mundorf R, Barnes W, Williams J. Fujii M. Effect of pulsed ultrasound versus placebo on musclesoreness perception and muscular performance. Scandinavian four nal of Rehabilitation Medicine 1990;22:199-205.

7 High DM, Howley ET, Franks BD. The effects of static igh DM, Howley ET, Franks BD. The effects of static stretching and warm-up on prevention of delayed-
muscle soreness. Res $Q$ Exerc Sport 1989;60:357-61.

8 Matthews JNS, Altman Dg, Campbell MJ, Royston P. Analysis of serial measurements in medical research. $B M \mathcal{F}$ Analysis of serial 